\title{
The First Young Brown Dwarf in the Serpens Cloud
}

\author{
Jean-Louis Monin \\ Observatoire de Grenoble Laboratoire d'Astrophysique BP 5338041 \\ Grenoble cedex
}

Emmanuel Caux and Alain Klotz

CESR CNRS-UPS, BP 4346, 31028 - Toulouse cedex 04, France

Nicolas Lodieu

Astrophysikalisches Institut Potsdam, An der Sternwarte 16, D-14482
Potsdam, Germany

\begin{abstract}
We report the discovery of the first young brown dwarf in the Serpens cloud (BD-Ser 1). It is obscured by more than ten magnitudes of visual absorption as indicated by near infrared (NIR) photometric survey at the NTT and confirmed by NIR spectroscopy at the VLT. We estimate the mass of this brown dwarf to be $\mathrm{M} \sim 0.05 \mathrm{M}_{\odot}$ and its age to be $\sim 3.5 \mathrm{Myr}$. Available NIR indices in the literature (designed for field brown dwarfs) fail to provide its current spectral type but using a model they correctly determine its future spectral type to be T. This is the first young brown dwarf ever found deeply embedded in the Serpens star formation region
\end{abstract}

\section{Introduction}

The identification of a significant number of young brown dwarfs in a well studied star formation region will allow to constrain the IMF down to the substellar regime and will help us answer to fundamental questions relative to star formation: is the IMF universal ? Does it keep rising in the substellar regime? How is the brown dwarf formation process connected to the one of solar type stars? Do the brown dwarfs present the same spatial distribution than normal stars in the cloud? Deep photometric and spectroscopic observations can help answer these questions.

In search for this young substellar population, we have performed a very deep photometric NIR survey in the Serpens cluster, one of the nearest active star forming regions in our Galaxy (Kaas 1999). From this survey, and using recent brown dwarf models (Baraffe et al. 1998), we have selected the best brown dwarf candidates to confirm their status using the NIR spectrometer ISAAC on the VLT. In this paper, we report the discovery of the first young $(\sim 3.5 \mathrm{Myr})$ brown dwarf associated with the Serpens cluster, we present its physical parameters and we discuss them against its spectral type. 


\section{Observations}

The photometric observations were carried out with SOFI in the $\mathrm{J}, \mathrm{H}$ and $\mathrm{Ks}$ photometric bands at the ESO 3.5-meter NTT in La Silla (see Lodieu et al., 2002 for details). Our JHKs results were translated from instrumental magnitudes to the standard JHK system using calibration stars measurements and checking the magnitudes of the brightest stars against previous studies (Eiroa and Casali 1992; Giovannetti et al. 1998). The bulk of our photometric uncertainties results from this translation.

In order to select brown dwarfs candidates, we placed our measures in a J$\mathrm{H} / \mathrm{H}-\mathrm{K}$ diagram. Possible traps in the selection of candidates from photometric observations come from extinct A-stars that mimic late $\mathrm{M}$ dwarfs with $\mathrm{A}_{V}=0$. From the NTT/SOFI photometric observations, a set of 20 brown dwarf candidates were selected for further spectroscopic investigations. The best candidate was found to lie along a reddening vector of $A_{V}=10.7$ from the dwarf end of the main-sequence locus in the J-H/H-K colour-colour diagram.

The spectroscopic observations were carried out at the ESO 8.2-meter Very Large Telescope Unit 1 (VLT Antu) on Paranal with the short wavelength (SW) channel of the NIR Spectrometer and Array Camera ISAAC (Moorwood and Spyromilio, 1997). We used the SW long slit low-resolution mode and the J, SH and SK ESO spectrometric filters $\left(R_{\lambda} \sim 500\right.$ for a $1^{\prime \prime}$ slit $)$. The resulting composite spectrum of BD-Ser 1 was obtained after airmass and absorption correction and cross calibration of the J, SH and SK spectral bands.

\subsection{Results}

The selection of BD-Ser 1 for subsequent spectroscopic observations was made essentially from its position in NIR colour-magnitude and colour-colour diagrams, using evolutionary tracks computed for young ( $\leq 10 \mathrm{Myr}$ ) brown dwarfs by Baraffe et al. (1998). The photometry of BD-Ser 1 is compatible with that of a $0.05 \mathrm{M}_{\odot}, 3.5 \mathrm{Myr}$ old deeply embedded $\left(\mathrm{A}_{V}=10.7\right)$ brown dwarf. However, the J-K error bar prevents a precise estimation of its masse and age. We have therefore used supplementary information from its spectrum to improve this estimation.

We have used the theoretical spectra of Allard et al. (2001) computed for $\mathrm{T}_{\text {eff }}=2800 \mathrm{~K}$ and $\log g=4$, the closest physical parameters of those of a $0.05 \mathrm{M}_{\odot}, 3.5 \mathrm{Myr}$ old brown dwarf $\left(\mathrm{T}_{\text {eff }}=2850 \mathrm{~K}, \log g=3.75, \mathrm{~L}=1.4810^{-2} \mathrm{~L}_{\odot}\right.$, Baraffe et al. 1998) to confirm the physical parameters of BD-Ser 1 and reduce their uncertainties. The agreement between the observed spectrum and the theoretical one obscured by $\mathrm{A}_{V}=10.7$ is very good. In order to assess the young brown dwarf status of BD-Ser 1 , we have also considered a theoretical spectrum of an older object at the hydrogen burning limit $\left(10 \mathrm{Myr}, 0.075 \mathrm{M}_{\odot}\right)$. This last spectrum differs significantly from the observed one and we conclude that BD-Ser 1 is a $0.05 \pm 0.02 \mathrm{M}_{\odot}$ brown dwarf of $3.5 \pm 2 \mathrm{Myr}$. This age is consistent with the conservative value estimated by Kaas (1999) for the Serpens cluster. Together with its visual absorption $A_{V}=10.7$, this is a strong evidence that BD-Ser 1 is physically associated with the cloud and was formed within, at the same location and time than other young stars. 
We have also checked the spectrum of BD-Ser 1 for typical brown dwarf features as the $\mathrm{CO}$ bands at $2.3-2.38 \mu \mathrm{m}$ and the water absorption bands at $1.11-1.16 \mu \mathrm{m}, 1.35-1.45 \mu \mathrm{m}, 1.8-2.0 \mu \mathrm{m}$ and longward of $2.4 \mu \mathrm{m}$. We possibly detect the $\mathrm{NaI}$ doublet in absorption near $2.2 \mu \mathrm{m}$, and clearly the $\mathrm{Br} \gamma$ line in emission. Muzerolle et al. (1998) have found a tight correlation in T Tauri stars between the strength of the $\mathrm{Br} \gamma$ line and the accretion luminosity as measured from the hot continuum excess. This could suggest that BD-Ser 1 is accreting matter from a circumstellar disk.

\section{Physical parameters and spectral type}

Recent studies have linked near-infrared and far-red/optical indices to provide a framework for brown dwarf spectral type classification (Tokunaga \& Kobayashi 1999, Reid et al. 2001, Testi et al. 2001, and Martín 2001). We have applied these indices to the spectrum of BD-Ser 1 . All the indices but two give a result consistent with a brown dwarf of spectral type L0-L3. Given the effective temperature of the model $(2850 \mathrm{~K})$, the actual spectral type of BD-Ser 1 is more likely that of a late $\mathrm{M}$ dwarf, a value consistent with its youth. Moreover, when we compute the indices on the theoretical spectrum from Allard et al. (2001), the NIR indices also give a L0-L2 spectral type. This disagreement likely comes from the fact that these practical indices have been designed for (older) field brown dwarfs and are not valid for young objects as BD-Ser 1.

As a spectral type is more a "snapshot" of a global image of an object, we favour the physical parameters extracted from the model spectrum and stick to the $T_{\text {eff }}=2850 \mathrm{~K}$ description. Indeed, if the notion of spectral type can have a useful meaning for a Main-Sequence star because the hydrogen fusion locks it to a given observable physical parameter range for billions of years, it might be less useful for evolving objects like brown dwarfs.

Then, if we use the same physical parameters through the Allard et al. (2001) model and compute a theoretical spectrum for the same object at an age of $200 \mathrm{Myr}$, the resulting indices for this older brown dwarf give a spectral type $\mathrm{T}$, a value consistent with its mass. At such age, our object will become a "field" brown dwarf and it is normal that the NIR indices provide the correct snapshot description.

This behavior is nicely illustrated if we plot the estimated age and effective temperature of BD-Ser 1 on the evolutionary tracks in Fig. 8 of Burrows et al (2001). The point $(3.5 \mathrm{Myr} ; 2850 \mathrm{~K})$ falls on the Deuterium burning limit in the $\mathrm{M}$ spectral type region on the $0.04 M_{\odot}$ track. As the brown dwarf ages, assuming that it essentially keeps the same mass, BD-Ser 1 will evolve toward the $\mathrm{T}$ spectral type region. We conclude that BD-Ser 1 is a future "field" T-type brown dwarf.

\section{Conclusion}

We have performed a deep near-infrared imaging survey of a 50 square arcminutes region in the Serpens cloud with SOFI on the NTT. This survey presents a deep detection limit of $\mathrm{M}_{K}=18.6$, deep enough to probe the substellar domain 
of the observed field despite its relatively high internal extinction. Among thousands of stars detected, 20 brown dwarf candidates were selected for follow-up near-infrared spectroscopic observations performed using ISAAC at the VLT. These observations allowed to confirm the brown dwarf status of at least one of our candidates. According to evolutionary tracks for brown dwarfs, we estimate the age of this object to be $3.5 \pm 2 \mathrm{Myr}$ and its mass to be $0.05 \pm 0.02 \mathrm{M}_{\odot}$. Given all the physical parameters that we can extract from the comparison with a theoretical spectra, it appears less useful to use a template spectral type to characterise the object. NIR indices designed for field brown dwarfs classify BD-Ser 1 as an early L type dwarf, when its effective temperature corresponds more probably to a late $\mathrm{M}$ dwarf. The use of an evolutionary model reconciles these two estimations as its future spectral type (at $t \geq 200 \mathrm{Myr}$, when BD-Ser 1 become a field dwarf) will be $\mathrm{T}$. This is the first young brown dwarf ever found deeply embedded in the Serpens star formation region.

\section{References}

Allard F., Hauschildt P.H., Alexander D.R. et al. 2001, ApL 556, 357

Baraffe I., Chabrier G., Allard F. \& Hauschildt P.H. 1998 A\&A 337,403

Burrows A., Hubbard W.B., Lunine J. I. \& Liebert J., 2001, Rev. Mod. Phys. 73,719

Eiroa C. \& Casali M.M., 1992 A\&A 262, 468

Giovannetti P., Caux E., Nadeau D. \& Monin J.-L., 1998 A\&A 330, 990

Kaas A.A. 1999, AJ 118, 558

Lodieu N., Caux E. Monin J.-L. \& Klotz a., 2002, A\&A 383, L15

Martín E.L., 2001, in Ultracool Dwarfs: New Spectral Types L and T, H.R.A. Jones and I.A. Steele (eds), Springer Verlag, p.153

Moorwood A., \& Spyromilio, J., 1997, The Early Universe with the VLT. Edited by Jacqueline Bergeron. Berlin : Springer, 1997., p.21

Muzerolle J., Hartmann L. \& Calvet N. 1998, AJ 116, 2965

Reid I.N., Burgasser, A. J., Cruz, K. L. et al., 2001, AJ 121, 1710

Testi L., D'Antona F., Ghinassi F. et al., 2001 ApJ 552, L147

Tokunaga A. \& Kobayashi N., 1999, AJ 117, 1010 